\title{
Modelling and interpreting optical spectra of galaxies at $\mathbf{R}=10000$
}

\author{
A. Lançon*, P. Ocvirk*, D. Le Borgne ${ }^{\dagger}$, C. Pichon**, P. Prugniel ${ }^{\ddagger}$, M. \\ Fioc $^{* *}$, B. Rocca-Volmerange ${ }^{* *}$ and C. Soubiran ${ }^{\S}$ \\ * Observatoire de Strasbourg (UMR 7550), Strasbourg, France \\ ${ }^{\dagger}$ Dept. of Astronomy \& Astroph., University of Toronto, Canada \\ ${ }^{* *}$ Institut d'Astrophysique de Paris, France \\ $¥$ Observatoire de Lyon, France \\ $\S$ Observatoire de Bordeaux, France
}

\begin{abstract}
One way to extract more information from the integrated light of galaxies is to improve the spectral resolution at which observations and analysis are carried out. The population synthesis code currently providing the highest spectral resolution is PÉGASE-HR, which was made available by $\mathrm{D}$. Le Borgne et al. in 2004. Based on an empirical stellar library, it provides synthetic spectra between 4000 and $6800 \AA$ at $\lambda / \delta \lambda=10000$ for any star formation history, with or without chemical evolution. Such a resolution is particularly useful for the study of low mass galaxies, massive star clusters, or other galaxy regions with low internal velocity dispersions.

After a summary of the main features of PÉGASE-HR and comparisons with other population synthesis codes, this paper focuses on the inversion of optical galaxy spectra. We explore the limits of what information can or can not be recovered, based on theoretical principles and extensive simulations. First applications to extragalactic objects are shown.
\end{abstract}

\section{INTRODUCTION}

Models for the formation of galaxies and of their internal structures become more and more complex, triggering rapid progress in several related fields of astronomy. Observations are improving (galaxy samples, spectral coverage, spatial and spectral resolution), population synthesis models are being developed further, and inversion methods are being investigated with the aim of optimizing the extraction of information from the data.

In the field of population synthesis, one direction of progress goes towards higher spectral resolutions. Resolving powers of the order of $\mathrm{R}=\lambda / \delta \lambda=2000$ are now being used very widely for galaxy observations. As already demonstrated with low resolution spectrophotometric indices, such spectra allow us to break the age-metallicity degeneracy in the case of single stellar populations. $R=2000$ is also adequate for the study of the kinematics of massive galaxies, for which internal velocity dispersions of order $100 \mathrm{~km} . \mathrm{s}^{-1}$ smooth out intrinsically finer lines.

Even higher spectral resolutions are needed to fully exploit the information present in the light of low mass galaxies, or of regions of galaxies with small internal velocity dispersions such as nuclear star clusters, low mass bulges, galaxy disks or bars. In the spectra of such objects, a wealth of strong and weak lines give access, at least 
in principle, to very detailed information about the abundances and kinematics of the brightest components of the stellar populations.

This paper summarizes fundamental and practical issues related to the synthesis and analysis of galaxy spectra at $\mathrm{R} \simeq 10000\left(\delta v \simeq 30 \mathrm{~km} \cdot \mathrm{s}^{-1}\right)$, based on PÉGASE-HR, the only synthesis package currently available for such a purpose (D. Le Borgne et al. [5]). After a description of the package in Sect. 2, we discuss intrinsic properties of the resulting single stellar population spectra (SSPs) and their effects on the analysis of galaxy spectra in terms of stellar age and metallicity distributions. We illustrate the quantitative effects of $\mathrm{R}$ and of the signal-to-noise ratio $(\mathrm{S} / \mathrm{N})$ on our ability to separate and characterize sub-populations. These results are based on regularized inversion methods that are flexible enough to handle complex non-linear problems such as age-velocity distributions. More details will be published by P. Ocvirk and collaborators.

\section{PÉGASE-HR}

PÉGASE-HR is an extension of the previously available population synthesis code PÉGASE.2, developed by M. Fioc \& B. Rocca-Volmerange (http: / / www . iap. fr / pegase/pegasehr/index.html). Both use identical inputs in terms of stellar evolution or yields, and if requested they ensure consistent evolution of the metallicity in the gas and stars. PÉGASE. 2 used the Basel library of stellar spectra (Lejeune et al. [6]) to produce low resolution spectra ranging from the UV to the near-IR (see RoccaVolmerange [10] for further extensions). PÉGASE-HR adds a zoom into the optical range, with the synthesis of galaxy spectra at $R \simeq 10000$ between 4000 and $6800 \AA$. The input high resolution stellar library results from careful interpolations between 1959 spectra of 1503 stars observed with the spectrograph ELODIE at Observatoire de Haute Provence (Prugniel \& Soubiran [9]). These spectra were initially observed at R=42 000, which warrants an excellent wavelength calibration and, in the smoothed spectra actually used, a well defined constant resolved element. The fundamental parameters of the library stars range from $3000 \mathrm{~K}$ to $60000 \mathrm{~K}$ in Teff, from -0.3 to 5.9 in $\log (\mathrm{g})$ and from -3.2 to +1.4 in $[\mathrm{Fe} / \mathrm{H}]$. SSP spectra are synthesized for $-2<[\mathrm{Fe} / \mathrm{H}]<+0.4$.

The Lick index predictions of PÉGASE-HR have been compared in detail with those of Bruzual \& Charlot ([3]: BC03), Thomas et al. ([11]: TMB03), Bressan et al. ([2]: BCT96) and Worthey \& Ottaviani ([15]: WO97), for ages larger than 1 Gyr. Indices agree well in general, and we will only highlight a few differences here (see [5] for details).

- As all empirical libraries, the ELODIE library is affected by the trends found in the abundance patterns of solar neighbourhood stars: metal deficient stars tend to show an enhancement in $\alpha$-element abundances. Only TMB03 apply a correction, and thus obtain somewhat smaller Mg indices for sub-solar SSPs.

- There are significant differences between authors in the line indices of old metal-rich populations. BC03 predict lower iron indices (Fe 5270, Fe 5335) than other groups in this regime, although they use the same evolutionary tracks as BCT96 and PÉGASEHR. WO97 produces a larger $\mathrm{Mg}_{\mathrm{b}}$ index than other authors. The main origin for these discrepancies is clearly the scarcity of high metallicity low temperature giants in any of 
the empirical libraries used by the groups considered here. An additional cause are the uncertainties in the fundamental parameters of these few stars. There is a clear need for improvement here.

- There are significant differences among the Balmer line indices of metal-poor populations $([\mathrm{Fe} / \mathrm{H}]<-0.6)$. Both the tracks and the input spectra are likely to contribute to the differences between PÉGASE-HR, WO97 and TMB03. The agreement between PÉGASE-HR and BC03, who use the same evolutionary tracks, is better, although at any given metallicity a given index would be interpreted as a slightly younger age when using BC03.

At $R \simeq 10000$, the passbands of the rectangular filters that define the Lick indices encompass many lines of a variety of elements. For the study of objects with internal velocity dispersions below $50 \mathrm{~km} . \mathrm{s}^{-1}$, one may consider indices with passbands as narrow as about $2 \AA$. It is then possible to isolate the age sensitive features from the metallicity sensitive ones, and to break the age-metallicity degeneracy within only a very small spectral range. A good example is found around $\mathrm{H} \gamma$ where two new indices, centered about $10 \AA$ apart, provide an excellent age-metallicity discrimination for single stellar populations observed at $\mathrm{S} / \mathrm{N} \geq 50$ (Le Borgne et al. [5]). At lower $\mathrm{S} / \mathrm{N}$, or more generally in the presence of composite populations, many indices must be combined. It then becomes interesting to work with the full spectra directly.

\section{STELLAR POPULATIONS FROM HIGH RESOLUTION OPTICAL SPECTRA}

The problem of extracting a star formation history from an integrated galaxy spectrum is notoriously ill-conditioned. There is a large amount of redundancy between the many fluxes of a spectrum, so that the number of independent pieces of information is smaller than it may look. On the other hand, the redundancy makes it possible to access this information at lower $\mathrm{S} / \mathrm{N}$ ratios (all $\mathrm{S} / \mathrm{N}$ ratios in the following are given per $\AA$ ). A fundamental cause of ill-conditioning is the slow and sometimes almost linear evolution of SSP spectra with age: good quality data is needed to distinguish a particular SSP from the linear combination of an older one and a younger one. The mean square differences between successive spectra, even on a logarithmic age scale, is not constant, and therefore time resolution is a function of age. Finally, degeneracies between age, metallicity and extinction set limits that depend on the wavelength coverage, resolution and $\mathrm{S} / \mathrm{N}$ of the data. As a result of ill-conditioning, there is a high risk of overinterpreting a galaxy spectrum. If one attempts to recover too many parameters, for instance too many independent age bins for the description of the star formation history, the solution will dominated (and not only contaminated) by the noise component of the data: it will be meaningless.

We have adapted non parametric inversion tools to the analysis of galaxy spectra (Ocvirk et al. [7]). The aim of this effort is to quantify the amount of information that can be extracted robustly from a galaxy observation, given a collection of SSP spectra. The tools developed are flexible. They can be used with any population synthesis predictions, 
and they can be adjusted to deal with a variety of linear or non-linear problems. Here we will focus on results obtained with the SSP spectra from PÉGASE-HR, which in particular allows us to discuss the role of spectral resolution at the wavelengths of these models.

\section{A test case : the star formation history at known $\mathrm{Z}$}

Before considering more complex situations, it is useful to examine one of the simplest inversion problems : the determination of the contributions of SSPs of various ages in a composite population of which the metallicity is known. The model envisaged to describe the data is a linear combination of the SSP spectra, which are normalized to a common mean flux and can be arranged to form the columns of a matrix (the "kernel"). The unknowns are the contributions of the various ages to the light (masses can be derived a posteriori using the mass-to-light ratios of the SSPs).

Even at high resolution, the singular values of the kernel span many orders of magnitude. This is a clear symptom of ill-conditioning, but it also illustrates the correlation between the noise patterns of SSPs of successive ages, which are slightly different combinations of a finite number of stellar spectra. For uncorrelated white noise corresponding to a S/N ratio of a few hundred (as appropriate for PÉGASE-HR), the singular values would not drop below a predictable minimum value. The number of singular values above this threshold corresponds to the number of independent pieces of information that can be recovered reliably. For PÉGASE-HR, this number is of about 6 . Therefore, one should either restrict the number of independent age bins to about 6 , or adopt what we call a regularized non-parametric approach, i.e. consider a larger number of age bins but add smoothness constraints. The non-parametric approach, which does not require a restrictive choice of bin limits, allows for more freedom in the shape of the solution. For example, peaks in the star formation history can be age-dated precisely. The smoothness constraints act as damping factors on the terms that would otherwise be dominated by noise. Simulations lead us to advocate the differential smoothing operators $\mathrm{D}_{2}$ or $\mathrm{D}_{3}$ defined in Pichon et al. [8]. The relative importance of the smoothing is an inversion parameter that can be set objectively (and thus automatically) in linear inversion problems, but still requires tuning based on simulations in more complex situations.

If the $\mathrm{S} / \mathrm{N}$ ratio of the galaxy data available are below a few hundred, the number of reliable pieces of information that can be recovered drops from 6 to smaller values. Stacking data to improve the $\mathrm{S} / \mathrm{N}$ ratio before inversion is advisable, but stacking solutions derived from noisy data does not lead to a more meaningful "average solution". The result would approach a predictable combination of singular solution vectors of the kernel, i.e. a "star formation history" that says something about intrinsic properties of the SSPs rather than about the galaxy under study.

Systematic simulations have been run to determine how well one can separate two dominant episodes of star formation using high resolution optical spectra. We define the time resolution as the shortest separation between the episodes that still leads to a clear separation between the two. This time resolution is found to improve with increasing $\mathrm{S} / \mathrm{N}$, stabilizing around $0.4 \mathrm{dex}$ at $\mathrm{S} / \mathrm{N}=200$ (0.8-0.9 dex at $\mathrm{S} / \mathrm{N}=20)$. Spectral resolution, 
between $\mathrm{R}=1000$ and $\mathrm{R}=10000$, has comparatively little impact. The random errors on the mean age of the episodes also decrease with increasing S/N, ranging from about $25 \%$ at $\mathrm{S} / \mathrm{N}=20$ to about $5 \%$ at $\mathrm{S} / \mathrm{N} \simeq 200$ (only cases in which a clear separation was achieved are counted; no systematic errors due, for instance, to errors in the stellar libraries or the evolutionary tracks are accounted for).

Another linear problem is obtained when the model adopted for a galaxy spectrum is a free linear combination of SSP spectra of different ages and metallicities. Then, solutions are two-dimensional distributions in the age-metallicity plane. The age-metallicity degeneracy becomes a major issue here. The study of the singular values of the kernel of this inversion problem shows that the number of independent pieces of information that can be recovered is larger than in the case of a single known metallicity, but not twice as large. For a single stellar population, the mean age and metallicity can be recovered precisely, but as a result of (inevitable) smoothing a broader distribution is found, that grossly aligns along the age-metallicity degeneracy line. Recovering useful distributions in the case of more complex star formation histories is in many cases impossible at $\mathrm{S} / \mathrm{N}$ ratios compatible with the PÉGASE-HR SSPs (i.e. a few hundred); and such S/N ratios are difficult to reach in galaxy observations. It is more reasonable to consider the restricted problem of a single-valued age-metallicity relation, which, however, is a nonlinear problem (see below).

In the cases discussed above, different approaches may be taken in dealing with the continuum of the spectra. If the observations provide a reliable flux calibration and if extinction corrections can be made (for example on the basis of emission line measurements), the continuum can be used. Otherwise, the inversion has to allow for some freedom in the shape of the continuum. The problem becomes non-linear, and is considered explicitely in the next section. A few conclusions of such experiments are relevant to the current section. The constraints on the age distribution of the stars at a given metallicity are present in the lines, and can be found even when the continuum is unreliable. The mean age and metallicity determination of a single burst population can also be recovered without exploiting the continuum (as expected from the literature on Lick indices). It seems however that the direction of the age-metallicity degeneracy depends somewhat on whether or not the continuum is considered a relevant constraint. If it is, the metallicity sensitivity parameter $\operatorname{dlog}($ age $) / \operatorname{dog}(Z)$ (defined via SSPs as an analog of the sensitivity parameter of Worthey [14]) is found to be smaller than in the opposite case.

\section{More realistic, non linear problems}

The issues of metallicity and continuum correction raised above have shown the need for non-linear analyses. When metallicity is allowed to depend on age and when the continuum is given some freedom, the model equation is :

$$
F_{\lambda}=\int_{t_{o}}^{t_{f}} \Lambda(t) B(\lambda, t, Z(t)) f(\lambda, \eta) \mathrm{d} t
$$



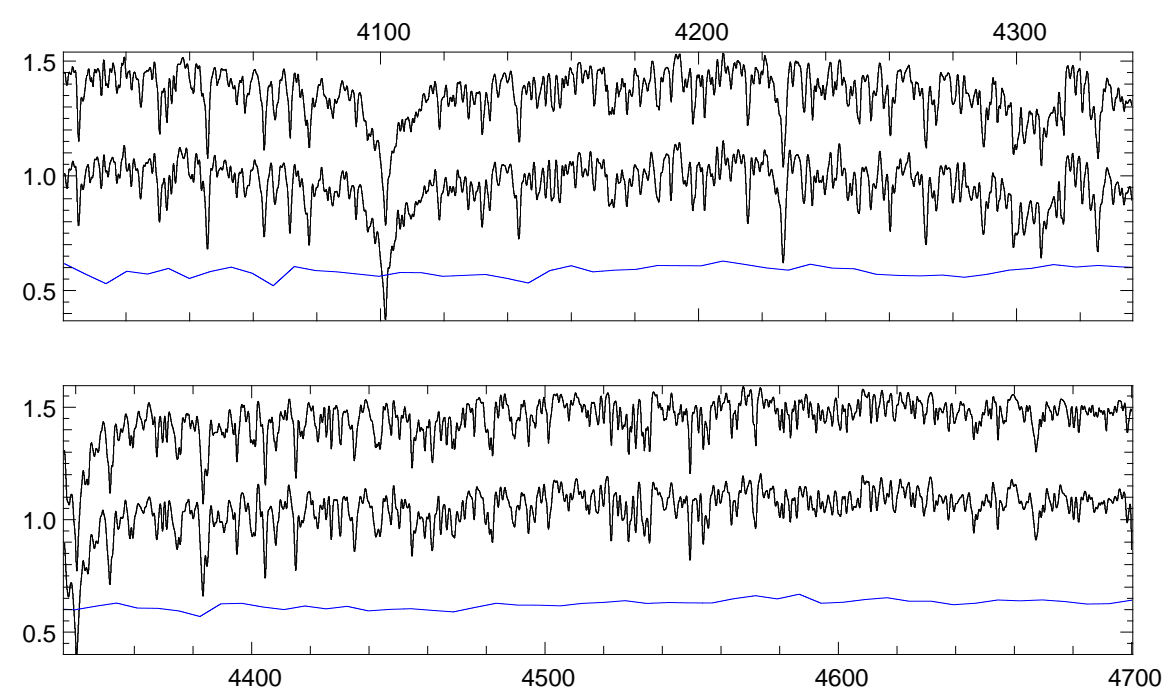

FIGURE 1. ESO/VLT/UVES spectrum of the nuclear cluster of NGC 300 (middle curve) and PÉGASEHR fit (top curve; a positive shift was applied for clarity). To account for unknown flux calibration errors in the data (and extinction), the inversion algorithm also searches for continuum adjustments. The resulting curve shows that only very small corrections are needed (bottom; a negative shift was applied for clarity).

where $F_{\lambda}$ is the galaxy spectrum, $B(\lambda, t, Z(t))$ is an SSP spectrum at age $t$ and metallicity $Z(t), \Lambda(t)$ is the contribution of this SSP to the light of the galaxy, and $f(\lambda, \eta)$ is some smooth function of wavelength that modifies the continuum (with one or several parameters $\eta$ ). Within this framework, we have repeated the separation experiments described in the linear case above. We find that time resolution is significantly worse than in the case of a known metallicity. It levels at 0.8 dex for $\mathrm{S} / \mathrm{N} \geq 100$, starting from values around $1.5 \mathrm{dex}$ at $\mathrm{S} / \mathrm{N} \simeq 20$. However, the actual ages of the two star formation episodes, when these are well separated, are recovered essentially as precisely as in the single metallicity case. The metallicities of the two episodes are also recovered properly. The random errors on these metallicities are below 0.06 dex for $\mathrm{S} / \mathrm{N}>50$ (again, no systematic errors are included here ${ }^{1}$ ). They depend on $\mathrm{S} / \mathrm{N}$ and on the spectral resolution, and $\mathrm{R} \geq 2500$ is recommended.

An application to observations of the nuclear cluster of the SAd-type galaxy NGC 300 is shown in Fig. 1 (reduced data kindly provided by C.J. Walcher). Using wavelengths between 4000 and $4700 \AA$, we find a best fit star formation history with two main star formation episodes (Ocvirk et al., in preparation). One is younger than $1 \mathrm{Gyr}$, the other is much older and has a lower metallicity. It is interesting to note that the younger episode is found to be dominant by Walcher et al. [12], who used a bluer part of the spectrum (between about 3800 and $4500 \AA$ ) and single-age BC03 models at solar metallicity. It is expected that the younger component would have a stronger contribution at shorter wavelengths. The presence of an old component would have a strong impact on the

\footnotetext{
${ }^{1}$ Note that it remains to be determined how the errors depend on the slope of the age-metallicity relation used to construct the mock data.
} 

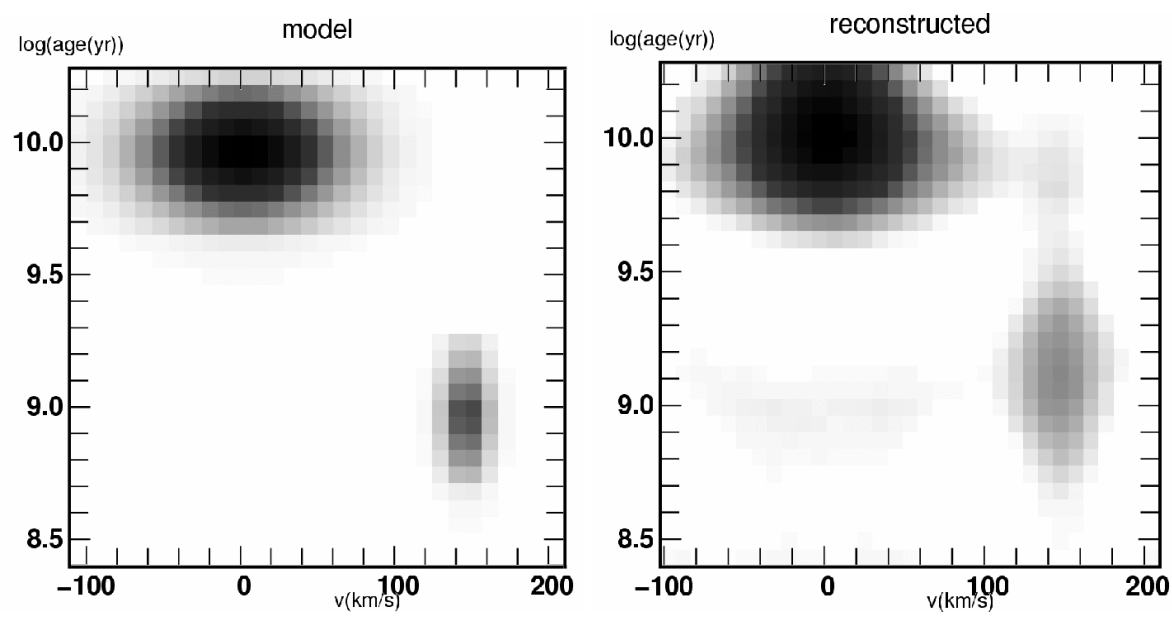

FIGURE 2. The use of high resolution optical spectra to search for age-velocity distributions. In this test case, the model (left) consists of an old component with a high velocity dispersion (for example a bulge) and a younger component at an offset velocity (for example a disk component). The main two-dimensional structure is recovered (right).

interpretation of the dynamical mass of the cluster (Walcher et al. [13]). However, we stress that our results are preliminary. The metallicity issue as well as other sytematics need to be considered very carefully before final statements can be made. The treatment of the continuum differs between authors, and there may be differences in the detailed treatment and spatial coverage of the data that were used. P. Ocvirk is in the process of testing how accurately one can recover the star formation histories of mock galaxies who's spectra are synthesized with BC03 SSPs and analysed with PÉGASE-HR. In any case, the example of NGC 300's central cluster shows the quality of PÉGASE-HR fits, and tends to support the existence of multiple star formation episodes in some nuclear galaxy clusters, suggested by Böker et al. [1].

Non-linear inversion algorithms are also necessary when the stellar populations of a galaxy are to be described both in terms of their nature (age, metallicity) and in terms of their kinematics. With high resolution data of high quality, one may attempt to search for distributions in age and velocity simultaneously. This may, for instance, help disentangling the properties of bulges and inner galaxy disks in the region of overlap. In this framework, the model spectrum for a region of a galaxy is a linear combination of single stellar populations, each of which is allowed to have a specific velocity distribution. The solution searched for is a two dimensional distribution in age-velocity space. The tools developed by Ocvirk et al. [7] allow us to tackle this problem, with specific smoothness constraints. Figure 2 illustrates preliminary results. We are in the process of determining more systematically which configurations are favourable or prohibitive for this type of inversion problem. In the mean time, PÉGASE$\mathrm{HR}$ is being used in the more traditional approach, which assumes that one velocity distribution adequately applies to all the stars that contribute significantly to the light. Chilingarian et al. [4] are analysing a sample of dwarf ellipticals in the Virgo clusters this way, using observations with the integral field spectrograph MPFS on the Russian $6 \mathrm{~m}$ telescope. Their velocity maps indicate decoupled cores or disks in a few objects. If 
the age or metallicity difference between these cores and the outer regions are sufficient, age-velocity inversions should provide interesting constraints on their formation history.

\section{Conclusions}

High resolution, high $\mathrm{S} / \mathrm{N}$ optical spectra in good agreement with observations of galaxies can be synthesized with PÉGASE-HR between 4000 and $6800 \AA$. While the $\mathrm{S} / \mathrm{N}$ ratio (per $\AA$ ) of the data is the main factor determining what resolution in stellar ages can be achieved, the spectral resolution is critical to improve the age-metallicity separation at these wavelengths and to study stellar kinematics. Regularized non-parametric inversion tools have been developed to analyse these (or other) data. When the problem is set properly and the data have a high $\mathrm{S} / \mathrm{N}$, the random errors of the solutions are often smaller than the systematic differences expected from the use of different population synthesis codes. Future progress should include an improvement of the stellar spectral libraries in certain parts of the Hertzsprung-Russell diagram (cool giants, hot stars), and the simultaneous analysis of high resolution spectra and lower resolution panchromatic energy distributions.

\section{ACKNOWLEDGMENTS}

We are grateful to J. Walcher for making reduced UVES spectra available to us. We thank the organizers for a very useful and well organized meeting.

\section{REFERENCES}

1. Böker T., van der Marel R., Gerssen J., Walcher J. et al. 2003, Proc. of SPIE 4834, 57

2. Bressan A., Chiosi C., Tantalo R. 1996, A\&A 311, 425 (BCT96)

3. Bruzual G., Charlot S. 2003, MNRAS 344, 1000 (BC03)

4. Chilingarian I., Prugniel P., Sil'chenko O., Afanasiev V. 2005, in preparation

5. Le Borgne D., Rocca-Volmerange B., Prugniel P., Lançon A. et al. 2004, A\&A 425, 881

6. Lejeune T., Cuisinier F., Buser R. 1998, A\&AS 130, 65

7. Ocvirk P., Pichon C., Lançon A., Thiébaut E. 2004, in "The Spectral Energy Distribution of GasRich Galaxies: Confronting Models with Data", Heidelberg, 4-8 Oct. 2004, eds. C.C. Popescu \& R.J. Tuffs, AIP Conf. Ser., in press

8. Pichon C., Siebert A., Bienaymé O. 2002, MNRAS 329, 181

9. Prugniel P., Soubiran C. 2004, research note, astro-ph/0409214

10. Rocca-Volmerange B. 2004, in "The Spectral Energy Distribution of Gas-Rich Galaxies: Confronting Models with Data", Heidelberg, 4-8 Oct. 2004, eds. C.C. Popescu \& R.J. Tuffs, AIP Conf. Ser., in press

11. Thomas D., Maraston C., Bender R. 2003, MNRAS 339, 897 (TMB03)

12. Walcher C.J., Häring N., Böker T. 2004, in Coevolution of Black Holes and Galaxies, L.C. Ho (ed.), Carnegie Astroph. Ser. 1, in press

13. Walcher C.J., van der Marel R.P., McLaughlin D. et al. 2005, ApJ (in press; astro-ph/0409216)

14. Worthey G. 1994, ApJS 95, 107

15. Worthey G., Ottaviani D.L., 1997, ApJS 111, 377 